\title{
ON THE COEFFICIENT PROBLEM FOR UNIVALENT FUNCTIONS
}

\author{
BY \\ MENAHEM SCHIFFER( $\left.{ }^{1}\right)$
}

1. Let us consider the family $F$ of univalent functions in a domain $D$ of the complex z-plane which contains the origin. We suppose all functions to be normalized by the conditions $f(0)=0, f^{\prime}(0)=1$ and define their local power series development

$$
f(z)=z+\sum_{n=2}^{\infty} a_{n} z^{n}
$$

The coefficient problem for the family consists in the search for bounds for the coefficients $a_{n}$ and for the set of points $\left\{a_{2}, a_{3}, \ldots, a_{n}, \ldots\right\}$ in the coefficient space which belongs to functions in $F$.

The problem of determining the functions in $F$, for which, say, the functional $\operatorname{Re}\left\{a_{n}\right\}$ takes its maximum, can be attacked by the method of variations and the extremal functions can be characterized by functional-differential equations [2], [4]. The general idea of this procedure is as follows: Let $w=f(z)$ map the domain $D$ onto the image domain $\Delta$ and let $\Gamma$ be the complement of $\Delta$ in the $w$-plane. To every point $w_{0} \in \Gamma$ one can find univalent functions in $\Delta$ of the form

$$
w^{*}=V[w]=w+\varepsilon w^{2} /\left(w-w_{0}\right) w_{0}^{2}+O\left(\varepsilon^{2}\right)
$$

such that $V(0)=0, V^{\prime}(0)=1$ and that the remainder term $O\left(\varepsilon^{2}\right)$ can be estimated uniformly in each closed subdomain of $\Delta$. The combined mapping function

$$
f^{*}(z)=V[f(z)]=f(z)+\varepsilon f(z)^{2} /\left(f(z)-w_{0}\right) w_{0}^{2}+O\left(\varepsilon^{2}\right)
$$

is then a competing function in $F$ with the $n$th coefficient

$$
a_{n}^{*}=a_{n}-\varepsilon \frac{1}{w_{0}^{3}} P_{n}\left(\frac{1}{w_{0}}\right)+O\left(\varepsilon^{2}\right)
$$

where $P_{n}(t)$ is a polynomial of degree $(n-2)$ in $t$ defined by the generating function

$$
\frac{f(z)}{1-t f(z)}=\sum_{n=1}^{\infty}\left[a_{n}+t P_{n}(t)\right] z^{n}
$$

We can easily express the coefficients of the polynomial

$$
P_{n}(t)=\sum_{\nu=1}^{n-1} A_{n, v} t^{\nu-1}
$$

Received by the editors May 15, 1967.

(1) This work was supported in part by Air Force contract AF 49(638) 1345 at Stanford University. 
in terms of the coefficients of

$$
f(z)^{n}=\sum_{k=n}^{\infty} a_{n, k} z^{k}
$$

Indeed, from (5) and (7) we infer

$$
A_{n, v}=a_{v+1, n}
$$

The condition that $f(z)$ be the function with the largest value of $\operatorname{Re}\left\{a_{n}\right\}$ in the family $F$ leads to the inequality

$$
\operatorname{Re}\left\{\varepsilon \frac{1}{w_{0}^{3}} P_{n}\left(\frac{1}{w_{0}}\right)\right\}+O\left(\varepsilon^{2}\right) \geqq 0
$$

for all admissible variations (2). The basic lemma of the calculus of boundary variations [2], [4] allows us then to infer that $\Delta$ is a slit domain whose boundary $\Gamma$ consists of analytic arcs with the differential equation

$$
\frac{w^{\prime}(\tau)^{2}}{w(\tau)^{3}} P_{n}\left(\frac{1}{w(\tau)}\right)+1=0
$$

if $\tau$ is a properly chosen curve parameter.

It can even be proved [3] that

$$
P_{n}(1 / w) \neq 0, \quad w \in \Gamma, w \neq \infty,
$$

so the boundary arcs cannot fork at any finite point.

It is the purpose of the present paper to prove that the extremal function $f(z)$ is such that

$$
a_{2, n} \neq 0
$$

Since, clearly, we have

$$
P_{n}\left(\frac{1}{w}\right)=a_{2, n}+\frac{a_{3, n}}{w}+\cdots+\frac{a_{n-1, n}}{w^{n-3}}+\frac{1}{w^{n-2}}
$$

we conclude from the differential equation (10) that the boundary $\Gamma$ has near infinity the asymptotic direction $e^{i \sigma}$ defined by

$$
e^{-i \sigma} a_{2, n}<0 \text {. }
$$

Hence the boundary component of the extremum domain which contains the point at infinity has an asymptotic direction there.

A consequence of this fact is that, in the case of univalent functions in the unit disc, the extremal domain is bounded by one single analytic arc ending with asymptote at infinity. This fact was first proved by Schaeffer-Spencer [1]. They derived it from a careful analysis of the coefficient body of univalent functions. In a recent Stanford thesis, F. T. Johnson has derived the same result from an analogous 
result for univalent meromorphic functions in the unit disc by a passage to the limit.

It seems that the fact $a_{2, n} \neq 0$ is proved here for the first time in the case of all extremum problems for univalent functions. It may be very helpful in the further analysis of the functional-differential equation.

2. To prove our assertions, we have to complement the variations (2) which single out finite boundary points $w_{0}$ by an additional variation near the point at infinity. Indeed, since the functions $f(z) \in F$ are, by assumption, regular in $D$, the point at infinity lies in $\Gamma$.

We consider a part $\Gamma^{\prime} \subset \Gamma$ which is located near infinity and which bounds a simply-connected domain $\Delta^{\prime}$ in the $w$-plane which contains the origin and has with respect to it the mapping radius $R$. That is, there exists a univalent function $w=\Phi(\zeta)$ in the disc $|\zeta|<R$ with $\Phi(0)=0, \Phi^{\prime}(0)=1$ which maps it onto $\Delta^{\prime}$. Clearly,

$$
w=\Phi(\zeta)=R \varphi\left(\frac{\zeta}{R}\right)=\sum_{\nu=1}^{\infty} A_{\nu} R^{-(\nu-1) \zeta^{\nu}}, \quad A_{1}=1
$$

where $\varphi(\eta)$ is a normalized univalent function in $|\eta|<1$. By shrinking the complement $\Gamma^{\prime}$ towards infinity, we can make $R$ as large as we wish.

We have the well-known estimates

$$
\left|A_{2}\right| \leqq 2, \quad\left|A_{3}\right| \leqq 3, \ldots, \quad\left|A_{n}\right| \leqq \text { en }
$$

Hence the inverse function

$$
\zeta=w-\frac{A_{2}}{R} w^{2}+\frac{2 A_{2}^{2}-A_{3}}{R^{2}} w^{3}+O\left(\frac{1}{R^{3}}\right)
$$

represents a univalent variation of $\Delta$ which allows a uniform estimate of the error term $O\left(1 / R^{3}\right)$ in every closed subdomain of $\Delta$.

We may introduce a more general variation by mapping $\Delta^{\prime}$ onto $|\zeta|<R$ and superimposing the additional mapping

$$
\zeta^{*}=\frac{\zeta}{\left(1-\left(e^{i \alpha} \zeta / R\right)\right)^{2}}=\sum_{\nu=1}^{\infty}\left(\frac{e^{i \alpha}}{R}\right)^{\nu-1} \nu \zeta^{\nu}
$$

of that unit disc with arbitrary real $\alpha$. Thus we have the new variations

$$
\zeta^{*}(w)=w+\frac{1}{R}\left(2 e^{i \alpha}-A_{2}\right) w^{2}+\frac{1}{R^{2}}\left(3 e^{2 i \alpha}-4 A_{2} e^{i \alpha}+2 A_{2}^{2}-A_{3}\right) w^{3}+O\left(\frac{1}{R^{3}}\right),
$$

and the functions

$$
\begin{aligned}
f^{*}(z)= & f(z)+\frac{1}{R}\left(2 e^{i \alpha}-A_{2}\right) f(z)^{2} \\
& +\frac{1}{R^{2}}\left(3 e^{2 i \alpha}-4 A_{2} e^{i \alpha}+2 A_{2}^{2}-A_{3}\right) f(z)^{3}+O\left(\frac{1}{R^{3}}\right)
\end{aligned}
$$

lie for all real $\alpha$ in the family $F$ and are competing functions. 
The $n$th coefficient of the varied function has the form

$$
a_{n}^{*}=a_{n}+\frac{1}{R}\left(2 e^{i \alpha}-A_{2}\right) a_{2, n}+\frac{1}{R^{2}}\left(3 e^{2 i \alpha}-4 A_{2} e^{i \alpha}+2 A_{2}^{2}-A_{3}\right) a_{3, n}+O\left(\frac{1}{R^{3}}\right) .
$$

The extremum condition on $f(z)$ leads to the inequality

$$
\operatorname{Re}\left\{\left(2 e^{i \alpha}-A_{2}\right) a_{2, n}+\frac{1}{R}\left(3 e^{2 i \alpha}-4 A_{2} e^{i \alpha}+2 A_{2}^{2}-A_{3}\right) a_{3, n}\right\} \leqq O\left(\frac{1}{R^{2}}\right)
$$

for large $R$ and every choice of $\alpha$.

3. From the original differential equation for $\Gamma$ we know that we may choose $\Gamma^{\prime}$ as an analytic arc with a specific asymptotic direction at infinity. In this case, the coefficient $A_{v}(R)$ will have the asymptotic form

$$
A_{\nu}(R)=\nu e^{i(\nu-1) \beta}+O\left(\frac{1}{R}\right),
$$

where $\beta$ depends on the asymptotic direction but not on $R$.

If $a_{2, n} \neq 0$, we infer from (22) and (23)

$$
\operatorname{Re}\left\{\left(e^{i \alpha}-e^{i \beta}\right) a_{2, n}\right\}=0 \text { for all } \alpha .
$$

This leads to a simple relation between $\operatorname{sgn} a_{2, n}$ and $\beta$ which is equivalent to (14) and does not give new information.

But suppose $a_{2, n}=0$. Now, the maximum condition (22) becomes

$$
\operatorname{Re}\left\{a_{3, n}\left(3 e^{2 i \alpha}-8 e^{i(\alpha+\beta)}+5 e^{2 i \beta}\right)\right\} \leqq 0
$$

for all real $\alpha$. We define the polynomial

$$
\Pi(\zeta)=(\zeta-1)(3 \zeta-5)=3 \zeta^{2}-8 \zeta+5
$$

and can bring (25) into the form

$$
\operatorname{Re}\left\{a_{3 . n} e^{2 i \beta} \Pi(\zeta)\right\} \leqq 0 \quad \text { for all }|\zeta|=1 .
$$

We use first the values $\zeta=e^{i \varepsilon}$ with small $\varepsilon$ and find

$$
\operatorname{Re}\left\{a_{3, n} e^{2 i \beta}\left(i \varepsilon-\frac{1}{2} \varepsilon^{2}+\cdots\right)(-2+3 i \varepsilon+\cdots)\right\} \leqq 0
$$

for all real $\varepsilon$. The lowest order term in $\varepsilon$ leads to the conclusion

$$
a_{3, n} e^{2 i \beta}=r, \quad r=\text { real }
$$

and we obtain from (28)

$$
r \cdot \operatorname{Re}\left\{-\varepsilon^{2}+O\left(\varepsilon^{3}\right)\right\} \leqq 0, \quad r \geqq 0 .
$$

Next, choose $\zeta=-1$ and since $\Pi(-1)=16$, we obtain from (27) and (29):

$$
16 r \leqq 0, \quad r \leqq 0 .
$$

Thus, from $a_{2, n}=0$ we are led to the conclusion

$$
a_{3, n}=0 \text {. }
$$


4. To find the first coefficient $a_{k, n}$ in (13) which does not vanish, we proceed as follows. Let

$$
w=p(\zeta)=\frac{\zeta}{(1-\zeta)^{2}}
$$

with the inverse function

$$
\zeta=p^{-1}(w)=\frac{(1+4 w)^{1 / 2}-1}{(1+4 w)^{1 / 2}+1}
$$

We construct the function

$$
\frac{1}{a} p\left[a p^{-1}(w)\right]=\frac{4 w}{\left[(1+a)+(1-a)(1+4 w)^{1 / 2}\right]^{2}}
$$

In the special case $a=-1$ we obtain

$$
-p\left(-p^{-1}(w)\right)=\frac{w}{1+4 w}=\sum_{n=1}^{\infty}(-4)^{n-1} w^{n} .
$$

In the case $a=e^{i \varepsilon}$ with small $\varepsilon$ we may develop the function (34) into a series of the small parameter $(1-a)$ and find

(36) $\frac{1}{a} p\left[a p^{-1}(w)\right]=w-\frac{8(1-a)}{(1+a)^{3}}\left((1+4 w)^{1 / 2}-1\right) w+48 \frac{(1-a)^{2}}{(1+a)^{4}} w^{2}+O\left((1-a)^{3}\right)$.

Observe that the function $\varphi(\eta)$ introduced in (15) tends with $R \rightarrow \infty$ toward the rational limit function

$$
\varphi_{\infty}(\eta)=e^{-i \beta} p\left(e^{i \beta} \eta\right)
$$

The inverse function (17) has the form

$$
\zeta=R \varphi^{-1}(w / R)
$$

and the composite variation (19) depending upon the parameter $\alpha$ appears as

$$
\zeta^{*}=V(w ; R, \alpha)=R e^{-i \alpha} p\left\{e^{i \alpha} \varphi^{-1}(w / R)\right\} .
$$

If we develop $V(w ; R, \alpha)$ into a power series in $w$ near $w=0$, we obtain

$$
V(w ; R, \alpha)=\sum_{\nu=1}^{\infty} h_{\nu}(R ; \alpha) \frac{1}{R^{\nu-1}} w^{\nu}
$$

Let us define

$$
V(w ; \infty, \alpha)=e^{-i \beta} \frac{1}{a} p\left[a p^{-1}\left(e^{i \beta} w\right)\right]=\sum_{\nu=1}^{\infty} h_{\nu}(\alpha) w^{\nu}, \quad a=e^{i(\alpha-\beta)} .
$$

It is evident that

$$
\lim _{R \rightarrow \infty} h_{\nu}(R, \alpha)=h_{\nu}(\alpha)
$$


We insert now the function $w=f(z)$ into the variation (39) and obtain the value

$$
a_{n}^{*}=a_{n}+\frac{a_{k, n}}{R^{k-1}} h_{k}(R, \alpha)+O\left(\frac{1}{R^{k}}\right)
$$

for the varied $n$th coefficient if $a_{k, n}$ is the first nonvanishing coefficient in (13).

The maximum condition for $f(z)$ with respect to $\operatorname{Re}\left\{a_{n}\right\}$ leads thus to the limit inequality

$$
\operatorname{Re}\left\{a_{k, n} h_{k}(\alpha)\right\} \leqq 0
$$

for all values of $\alpha$. We evaluate this condition for $a=e^{i \varepsilon}$ near $a=1$. We find from (36) and (41) the asymptotic formula

$$
h_{k}(\alpha)=-8 \frac{1-a}{(1+a)^{3}} 4^{k-1}\left(\begin{array}{c}
\frac{1}{2} \\
k-1
\end{array}\right) e^{i(k-1) \beta}+O\left((1-a)^{3}\right)
$$

if we assume $k>2$. Thus, since

$$
\operatorname{sgn}\left(\begin{array}{c}
\frac{1}{2} \\
k-1
\end{array}\right)=(-1)^{k}
$$

we derive from (44) the inequality

$$
\operatorname{Re}\left\{a_{k, n} e^{i(k-1) \beta}(-1)^{k} \frac{1-e^{i \varepsilon}}{\left(1+e^{i \varepsilon}\right)^{3}}\right\}+O\left(\varepsilon^{3}\right) \geqq 0,
$$

that is,

$$
\operatorname{Re}\left\{a_{k, n} e^{i(k-1) \beta}(-1)^{k} \frac{-i \sin \varepsilon / 2}{e^{i \varepsilon}(\cos \varepsilon / 2)^{3}}\right\}+O\left(\varepsilon^{3}\right) \geqq 0 .
$$

Since $\varepsilon$ can be chosen positive or negative, we find by considering the first order terms only

$$
a_{k, n} e^{i(k-1) \beta}=r, \quad r \text { real. }
$$

From the second order terms we read off

$$
(-1)^{k+1} r \cdot \varepsilon \sin (\varepsilon / 2)+O\left(\varepsilon^{3}\right) \geqq 0,
$$

that is,

$$
r(-1)^{k+1} \geqq 0 .
$$

Next, we choose $a=-1$. From (35) and (41) we find in this case

$$
h_{k}(\alpha)=e^{i(k-1) \beta}(-4)^{k-1} \text {. }
$$

Hence (44) becomes

$$
\operatorname{Re}\left\{a_{k, n} e^{i(k-1) \beta}(-1)^{k-1}\right\} \leqq 0 .
$$

By use of (48) we obtain

$$
r(-1)^{k-1} \leqq 0 .
$$


Combining $\left(49^{\prime}\right)$ with (52), we are forced to conclude

$$
r=0 .
$$

But we assumed $a_{k, n} \neq 0$ and ran into a contradiction.

Thus, the only noncontradictory assumption is $a_{2, n} \neq 0$ and our assertions are proved.

5. The variations near infinity, which were introduced in this paper, form an important additional set to discuss extremum problems involving univalent functions which do not take the value infinity. The considerations used above can be extended to any linear functional of the univalent function $f(z)$ and applied in the case of many more extremum problems.

\section{REFERENCES}

1. A. C. Schaeffer and D. C. Spencer, Coefficient regions for schlicht functions, Amer. Math. Soc. Colloq. Publ., Vol. 35, Amer. Math. Soc., Providence, R. I., 1950.

2. M. Schiffer, A method of variation within the family of simple functions, Proc. London Math. Soc. (2) 44 (1938), 432-449.

3. - On the coefficients of simple functions, Proc. London Math. Soc. (2) 44 (1938), $450-452$.

4. - Extremum problems and variational methods in conformal mapping, Proc. Internat. Congress Math., 1958, Cambridge Univ. Press, New York, 1960, pp. 211-231.

5. R. Courant, Dirichlet's principle, conformal mapping, and minimal surfaces, Interscience, New York, 1950; Appendix.

STANFORD UNIVERSITY,

Stanford, California 